\title{
CONSTRAINTS IN GENERAL RELATIVITY IN THE FORM OF MAXWELL EQUATIONS
}

A. UNT, V. UNT. OLDRELATIIVSUSTEOORIA SEOSEVORRANDID MAXWELLI VORRANDITE KUJUL

A. УНТ, В. УНТ, УРАВНЕНИЯ СВЯЗЕН ОБЩЕИ ТЕОРИИ ОТНОСИТЕЛЬНОСТИ В ФОРМЕ УРАВНЕНИИ МАКСВЕЛЛА

\section{(Presented by G. Kuzmin)}

The Einstein field equations are not independent since they satisfy the Bianchy identities and the integration of the equations consists of two separate problems. First, the solution of constraint equations for initial data. Secondly, the problem of time-development or evolution. The initial-value problem has been discussed in a great number of papers, including the survey by Y. Choquet-Bruhat and J. York [ $\left.{ }^{1}\right]$. Rigorous results have been achieved both in the study of compact cases and the systems in asymptotically flat space-time that are not losing energy via radiation.

In order to have an insight into the problem of radiative fields in asymptotically flat space-time we propose to consider the linearized Einstein equations with the sources in the form of electromagnetic radiation. The asymptotic behaviour that characterizes the energymomentum tensor of the electromagnetic field ${ }^{*} T_{E M}^{\mu v}$ is also characteristic of the nonlinear terms representing gravitational radiation.

In this paper we show that the linearized initial-value equations can be presented in the form of the second pair of the Maxwell equations, whereas the role of current $g^{\alpha}$ is played by the $T^{0 \alpha}$ components of the matter tensor. Then we demonstrate that by the application of the Hilbert coordinate conditions (harmonic coordinate conditions in linearized form), we get the full system of the Maxwell equations in the Lorentz gauge. This approach can also be used for having a thorough insight into the physical content of constraint equations.

The exact Einstein equations can be put down in the following form $\left[{ }^{2}\right]$ :

$$
U_{\mu}^{[v \sigma]}{ }_{, \sigma}-t_{\mu}{ }^{\nu}=16 \pi \gamma_{\mu} \mathcal{T}_{\mu}{ }^{\nu}
$$

The linearized form of these equations is

$$
\begin{gathered}
S_{[\mu \rho][v \sigma]}^{[\sigma \sigma}=16 \pi \gamma^{\mu \nu}, \\
S[\mu \rho][v \sigma] \equiv \eta^{\mu v} \gamma^{\rho \sigma}+\eta^{\rho \sigma} \gamma^{\mu v}-\eta^{\mu \sigma} \gamma^{\rho v}-\eta^{\rho v} \gamma^{\mu \sigma}, \\
\gamma^{\mu v} \equiv g^{\mu v}-\eta^{\mu v},
\end{gathered}
$$

where $\gamma$ is the gravitational constant, $g^{\mu v}$ is the density of a metric tensor and $\eta^{\mu \nu}$ is the Minkowski metric tensor; $T^{\mu v}$ is the flat space-time value of $\int^{\mu \nu}$ and $S^{[\mu \rho]]^{[v \sigma]}, \sigma}$ is the linearized form of $U^{v[\mu \sigma]}$. The indices

* Greek indices take the value $0,1,2,3$; Latin indices $-1,2,3$; $x^{0}$ denotes the time coordinate. 
in square brackets denote antisymmetrization. By the insertion of the definition formulae (2) into the equation (1), we get the well-known form of the linearized Einstein equations.

Let us define

$$
\begin{aligned}
F^{\mu \rho} & \equiv-S[\mu \rho][0 s]_{, s} / 4, \\
\mathcal{g}^{\mu} & \equiv \gamma^{\mu 0} .
\end{aligned}
$$

By the insertion of $v=0$ and the quantities (3) and (4) in the equations (1), we get the second pair of the Maxwell equations

$$
F^{\mu \rho}{ }_{, \rho}=-4 \pi \tilde{\jmath}^{\mu} \text {. }
$$

From the definitions (2) and (3) it follows that

$$
\begin{aligned}
& F^{m 0}=\left(\gamma^{m s}{ }_{, s}-\gamma^{00}{ }_{m}\right) / 4, \\
& F^{m r}=\left(\gamma^{0 m}{ }_{, r}-\gamma^{0 r}{ }_{m}\right) / 4 \equiv-\gamma^{0[m, r]} / 2 .
\end{aligned}
$$

Note that the indices after the comma are raised by the Minkowski metric. Define

$$
A^{\rho} \equiv\left(\gamma^{00}, \gamma^{0 i}\right) / 4
$$

In the Hilbert gauge $\gamma^{\mu \sigma}{ }_{, \sigma}=0, \gamma^{m s}{ }_{, s}=-\gamma^{m 0}{ }_{, 0}, F^{m 0}=\gamma^{0[0, m]} / 2$, and we have

$$
F \mu \rho=2 A[\rho, \mu] .
$$

Thus, the first pair of the Maxwell equations is the trivial consequence of the form (9) of the field tensor. The Lorentz condition follows from the definition formula (8) and the Hilbert condition $\gamma^{0 \sigma}{ }_{, \sigma}=0$.

Let us reproduce our results in three-dimensional form and underline the differences between the Maxwell and the Einstein theories. The «energy constraint» is

$$
\begin{aligned}
& \operatorname{div} \vec{E}=4 \pi \varrho, \\
& E^{m}=F^{m 0}, \quad \varrho \equiv \mathcal{\gamma}^{0}, \\
& E^{m}=\left(\gamma^{00, m}+\gamma^{m s}, s\right) / 4 .
\end{aligned}
$$

In the Hilbert gauge $\gamma^{m s}{ }_{, s m}=\gamma^{00}, 00, \operatorname{div} \vec{E}=\square \gamma^{00} / 4$, and we have

$$
\square \gamma^{00} / 4=4 \pi \mathrm{Q} \text {. }
$$

Now we have reached the most important point in our discussion. No kind of the known electric charges moves with the velocity of light. However, there do exist gravitational charges that move with the velocity of light, e. g. the energy (matter) of the zero rest mass-fields. For the large values of the radial coordinate in case of the bounded sources of the zero rest mass-fields, their energy density has the asymptotic form $\varrho=a(t-r, \vartheta, \varphi) / r^{2}$. In harmonic coordinates this form of $\varrho$ gives rise to unsatisfactory $\ln r / r$ asymptotic behaviour of $\gamma^{00}$. It is likely that an examination of the complete analogy with the electromagnetic case is not the best way to study the problems of gravitational radiation and harmonic coordinates are not the best coordinates in the treatment of the wave-zone problems.

The three-dimensional form of the «momentum constraints» is

$$
\operatorname{rot} \vec{H}=\frac{\partial \vec{F}}{\partial t}+4 \pi \vec{r},
$$

where $H_{i}=\varepsilon_{i k l} F^{k l}$ and $\varepsilon_{i k l}$ is the completely antisymmetric tensor. In 
contradistinction to the electromagnetic case, the equation (12) contains only the first time-derivatives of the gravitational field potentials, since the expression (11) in an unspecified gauge does not contain any time-derivatives.

It is easy to verify that the Maxwell equation

$$
\operatorname{div} \vec{H}=0
$$

is a trivial consequence of equality (7).

Assuming that the Hilbert condition $\gamma^{r \mu}, \mu=0$ is satisfied and considering the curl of this condition, we get

$$
\varepsilon_{l r k^{\prime}}\left(\gamma^{r s},{ }_{s k}+\gamma^{r 0}, 0 k\right)=0 .
$$

By the insertion of definitions (6) and (7), we get

$$
\operatorname{rot} \vec{E}=-\frac{\partial \vec{H}}{\partial t}
$$

Thus, we have proved that the energy and momentum constraints can be put down in the form of the second pair of the Maxwell equations, while equation (14) is the consequence of the coordinate condition $\gamma^{i \mu}{ }_{, \mu}=0$. We are of the opinion that this condition should not be used in the wave zone.

\section{REFERENCES}

1. Choquet-Bruhat, Y., York, J. The Cauchy problem in General Relativity and Gravitation (ed. by A. Held), 99. Plenum Press, New York-London, 1980.

2. Goldberg, J. Equations of Motion in Gravitation: an Introduction to Current Research (ed. by L. Witten), 126. John Wiley and Sons, Inc., New York, 1962.

Academy of Sciences of the Estonian SSR, Institute of Astrophysics and Atmospheric Physics
Received August 25, 1982 